\title{
Gastric ischemia following endoscopic submucosal dissection of early gastric cancer
}

\author{
Andreas Probst ${ }^{1}$, Bruno Maerkl ${ }^{2}$, Maximilian Bittinger $^{1}$, and Helmut Messmann ${ }^{1}$ \\ ${ }^{1}$ Department of Gastroenterology, Klinikum Augsburg, Stenglinstrasse 2, 86156 Augsburg, Germany \\ ${ }^{2}$ Institute of Pathology, Klinikum Augsburg, Augsburg, Germany
}

\begin{abstract}
Procedure-related complications of gastric endoscopic submucosal dissection (ESD) mainly include bleeding and perforation. Another complication is stricture formation after ESD close to the pylorus or close to the gastroesophageal junction. We report a case of an 86-year-old patient who developed extensive gastric ischemia after ESD for early gastric cancer. We suppose that the most likely reason for the ischemia was the submucosal injection of a large volume of a mixture of glycerol $(10 \%)$ and epinephrine (dilution 1: 50 000) that was used, in combination with the patient's underlying cardiovascular comorbidity. Gastric ischemia as a complication of gastric ESD has not been described previously. A conservative treatment approach seems justifiable. However, close endoscopic follow up for early recognition and treatment of a resulting stricture is recommended.
\end{abstract}

Key words Endoscopy · Early gastric cancer (EGC) · Endoscopic submucosal dissection (ESD) - Complications . Ischemia

\section{Introduction}

Endoscopic submucosal dissection (ESD) has gained wide acceptance in the treatment of early gastric cancers (EGCs) in Japan and also in the Western world recently. Its ability to resect lesions en bloc regardless of their size is the major advantage of ESD compared to conventional resection techniques. Procedure-related complications of gastric ESD have been described and mainly include bleeding and perforation [1]. Another complication reported after gastric ESD close to the pylorus or close to the gastroesophageal junction is stricture formation, especially when the resection

Offprint requests to: A. Probst

Received: September 16, 2009 / Accepted: December 25, 2009 exceeds $75 \%$ of the circumference [2]. We report a case of a patient who developed extensive gastric ischemia after ESD for EGC.

\section{Case report}

An 86-year-old-woman with a previous history of heart failure (New York Heart Association [NYHA] II) chronic atrial fibrillation, and chronic renal insufficiency was referred for ESD of an EGC. The EGC was a type IIa lesion with a diameter of $45 \mathrm{~mm}$ localized in the anterior gastric wall at the border of the corpus and antrum (Fig. 1). Biopsies had confirmed well-differentiated adenocarcinoma. Computed tomography (CT) had ruled out metastatic disease but had shown moderate arteriosclerosis of the aorta, the celiac trunk, and the superior mesenteric artery. ESD was performed using a hook-knife (KD-620LR; Olympus, Tokyo, Japan). After the injection of a mixture of glycerol $(10 \%)$, epinephrine (dilution 1: 50 000), and a small amount of indigo carmine, ESD was performed without recognizable problems. Procedure time was $3 \mathrm{~h}$ and a total amount of $360 \mathrm{ml}$ of the mixture had to be injected. Clipping was performed for large vessels in the resection ulcer after ESD. The resected specimen measured $60 \times 50 \mathrm{~mm}$ and confirmed $\mathrm{R} 0$ en-bloc resection of a well-differentiated EGC $45 \mathrm{~mm}$ in diameter. The lesion was restricted to the mucosa and showed no vascular or lymphatic invasion (Fig. 2). Sedation was performed with propofol. Continuous monitoring of electrocardiogram, blood pressure, and oxygen saturation showed normofrequent atrial fibrillation but no other pathological results during ESD and also postinterventionally until the next day. Twenty hours after the ESD, routine endoscopy revealed extensive ischemic necrosis of the gastric mucosa extending from the subcardial corpus to the prepyloric antrum circumferentially (Fig. 3). Abdominal tenderness and leukocytosis developed. After ruling out free 
intraabdominal air radiologically, therapy with antibiotics and proton-pump inhibitors was started and the patient recovered after a short period of fasting. CT scan to rule out thrombosis or thromboembolism was not performed after the ESD because of the uneventful course and the concomitant renal insufficiency. Four weeks after the ESD the patient presented with vomiting, and stricture of the gastric antrum had developed (Fig. 4). The stenosis could be managed successfully with repeated balloon dilatation, twice a week initially and once a week later for 6 weeks (Fig. 5).

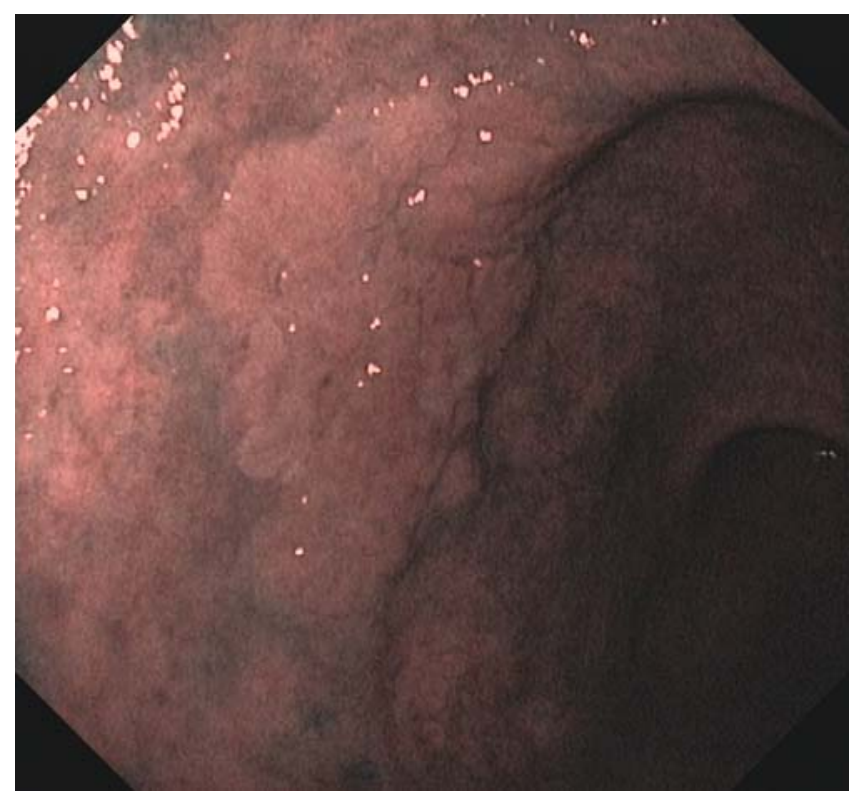

Fig. 1. Early gastric cancer (narrow band imaging)

\section{Discussion}

Known complications of ESD include bleeding, perforation, and stricture.

Even in large series gastric ischemia has not been observed as a complication of ESD so far.

Colonic ischemia has been reported as a complication after diagnostic colonoscopy [3] and also after sclerotherapy or cyanoacrylate injection for peptic ulcer bleeding [4-8]. In regard to the cyanoacrylate injection, thrombosis of large vessels was confirmed histologically

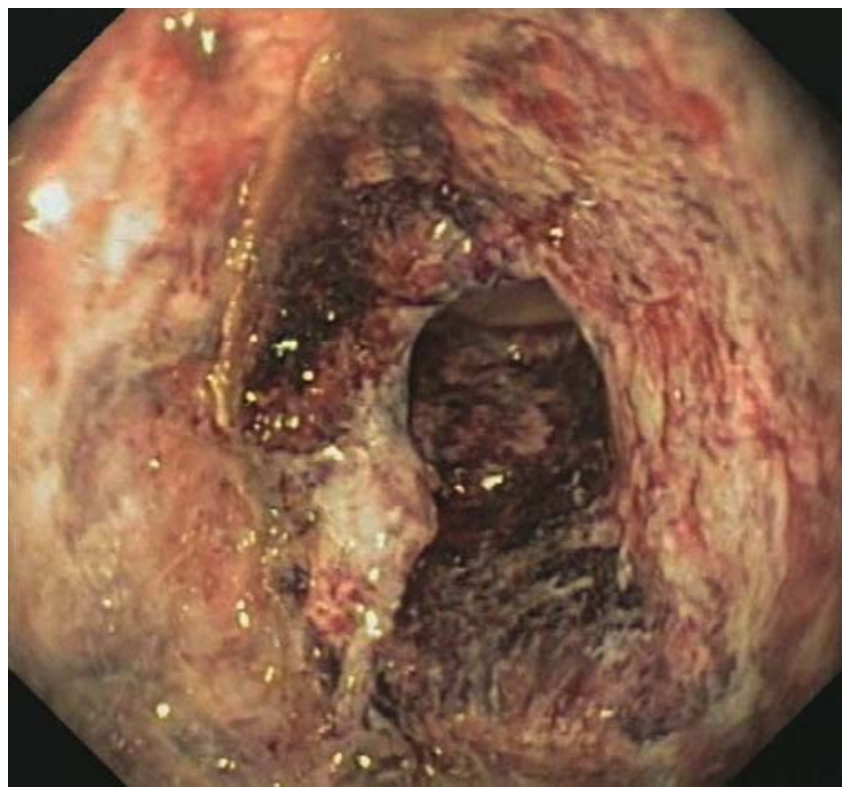

Fig. 3. Extensive gastric ischemic necrosis $20 \mathrm{~h}$ after endoscopic submucosal dissection (ESD)

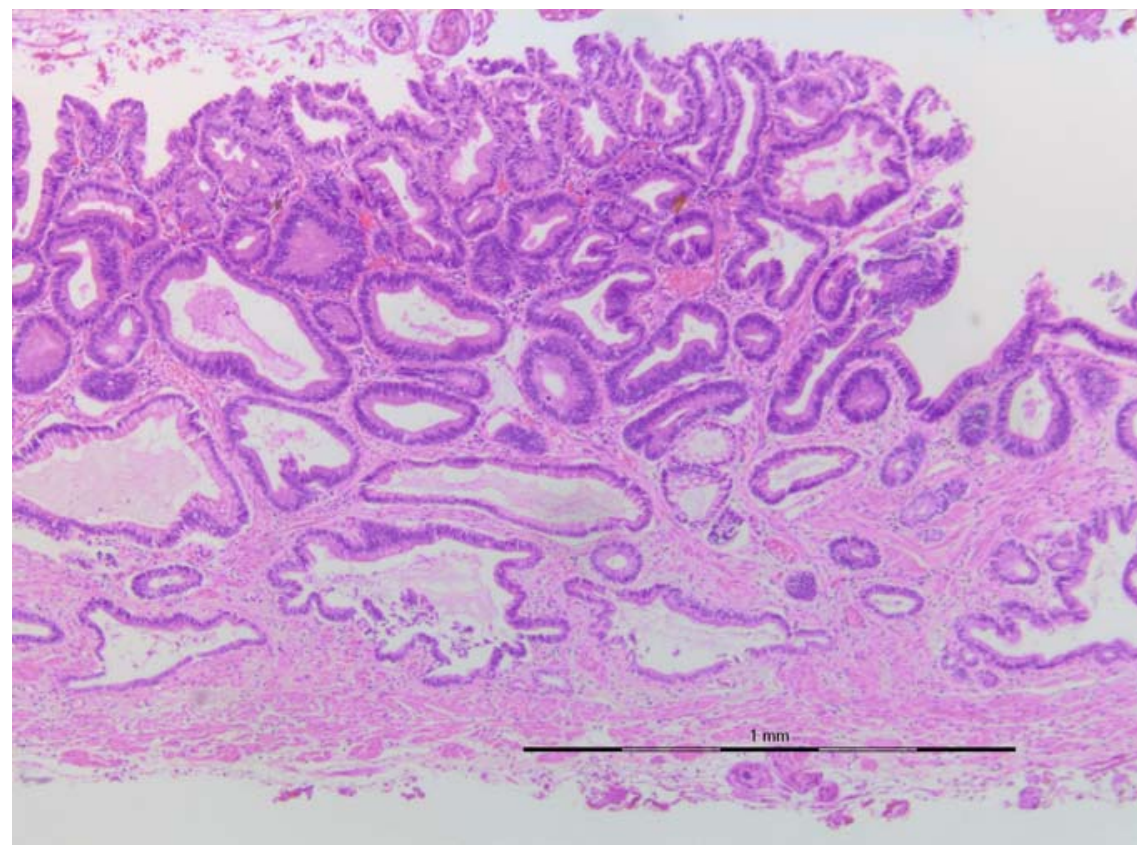

Fig. 2. Histology showing well-differentiated adenocarcinoma restricted to the mucosa. H\&E 


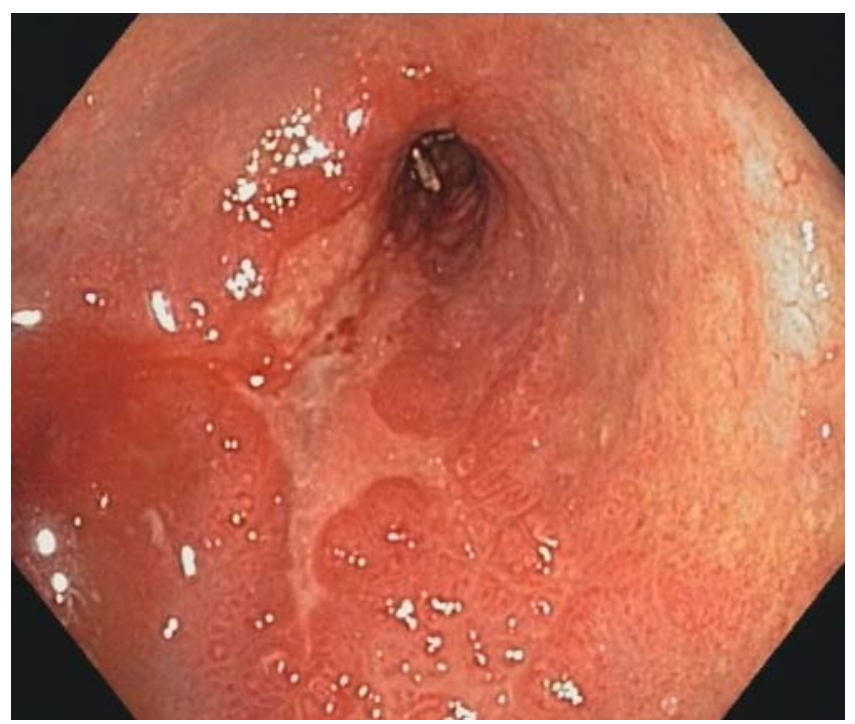

Fig. 4. Antral stenosis 4 weeks after ESD; note residual ischemic ulcers

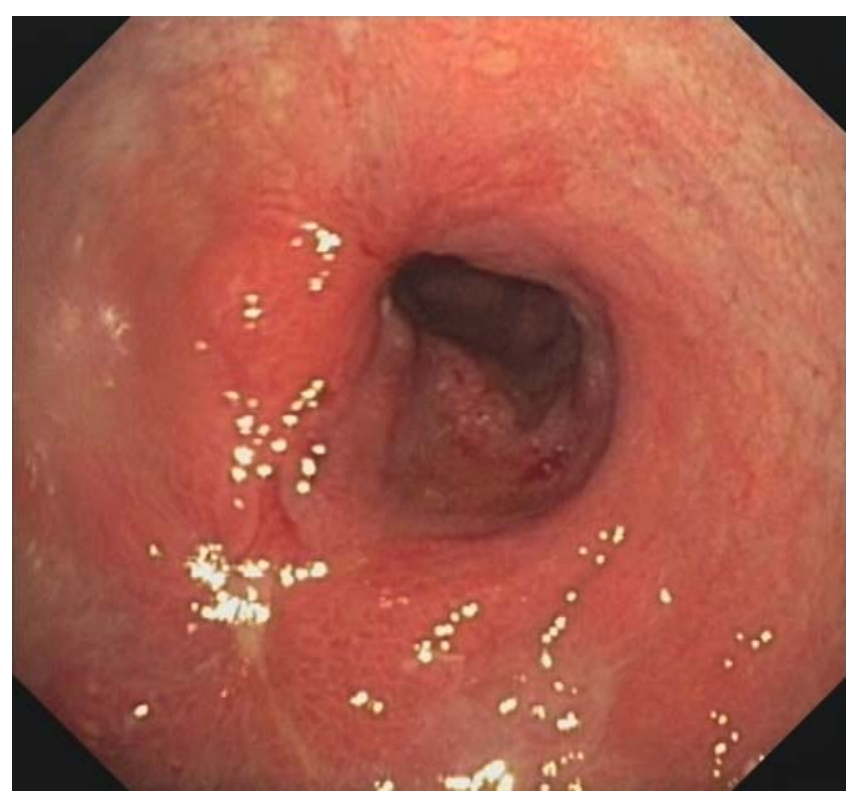

Fig. 5. Antral stenosis after repeated balloon dilatation

as the reason for the ischemia [8]. Accidental intraarterial injection of epinephrine, causing vasoconstriction, has been assumed by some authors to be a possible mechanism in the development of ischemia. Other factors discussed are compromised microcirculation [3] and anemia or hypotonia due to bleeding conditions. In our case the mechanism of the ischemia is not clear. First we supposed that the intraarterial injection of the epinephrine mixture was the most likely reason for the extensive gastric ischemia. Difficulties in lifting the lesion in the center, due to severe fibrosis and the large injection volume needed subsequently, may have promoted the danger of hitting larger gastric vessels within the gastric wall as well as on the gastric serosal surface. Another possible cause of the ischemia could have been the submucosal injection of a too large volume of epinephrine, causing vasoconstriction, or a too large volume of glycerol, causing mechanical compression of the submucosal tissue during the long procedure. However, the effect of one of these mechanisms alone is very unlikely to explain the wide and circular extension of the ischemia along both curvatures of the stomach which are fed by arcades of different gastric arteries. Although continuous monitoring had not shown hypotension or dips in oxygen saturation we cannot rule out a decrease in the local microcirculation or a local thromboembolism due to the underlying comorbidities of the patient such as heart failure, atrial fibrillation, and local arteriosclerosis. In summary, we suppose a combined mechanism of underlying vascular comorbidity, in combination with the large injection volume, to be the most likely reason for the gastric ischemia and its wide distribution in our patient.

Many reports on glycerol for submucosal injection show its effectiveness and safety $[9,10]$. However, there is no information so far regarding large injection volumes, especially in mixtures with epinephrine, and their possible risks.

Our case shows that the endoscopic submucosal injection of a high volume of glycerol mixed with epinephrine might cause ischemia, especially in a patient with underlying cardiovascular comorbidity and arteriosclerosis. Minimizing the injection volume and the epinephrine volume in these patients in particular could reduce this problem. A change to other substances such as hyaluronic acid or a further dilution of the epinephrine concentration could also be helpful to reduce the injection volume and to minimize the risk of this complication. Gastric ischemia as a complication of ESD has not been described previously. A conservative treatment approach seems justifiable. However, close endoscopic follow up for early recognition and treatment of a resulting stricture is recommended.

\section{References}

1. Gotoda T. Endoscopic resection of early gastric cancer. Gastric Cancer 2007;10:1-11.

2. Coda S, Oda I, Gotoda T, Yokoi C, Kikuchi T, Ono H. Risk factors for cardiac and pyloric stenosis after endoscopic submucosal dissection, and efficacy of endoscopic balloon dilation treatment. Endoscopy 2009;41:421-6.

3. Wheeldon NM, Grundman MJ. Ischemic colitis as a complication of colonoscopy. BMJ 1990;301:1080-1.

4. Dorta G, Michetti P, Burckhardt P, Gillet M. Acute ischemia followed by hemorrhagic gastric necrosis after injection sclerotherapy for ulcer. Endoscopy 1996;28:532. 
5. Goldberg PA, Krige JE. Fatal gastroduodenal necrosis after injection sclerotherapy for bleeding duodenal ulcer. J Clin Gastroenterol 1993;16:136-8.

6. Cappelii J, Dryjski J, Rahier I, Ramdani B, Lamy V, Moisse R. Another severe complication of sclerotherapy for bleeding peptic ulcer. Endoscopy 1993;25:431-2.

7. Loperfido S, Patelli G, La Torre L. Extensive necrosis of gastric mucosa following injection therapy of bleeding peptic ulcer. Endoscopy 1990;22:285-6.
8. Cheah WK, So J, Chong SM, Goh P. Duodenal ulcer perforation following cyanoacrylate injection. Endoscopy 2000;32:S23.

9. Uraoka T, Fujii T, Saito Y, Sumiyoshi T, Emura F, Bhandari P, et al. Effectiveness of glycerol as a submucosal injection for EMR. Gastrointest Endosc 2005;61:736-40.

10. Fujishiro M, Yahagi N, Kashimura K, Matsuura T, Nakamura M, Kakushima M, et al. Tissue damage of different submucosal injection solutions for EMR. Gastrointest Endosc 2005;62: 933-42. 